\title{
Programmable Self-Assembly-Unique Structures and Bond Uniqueness
}

\author{
Björn Högberg* and Håkan Olin \\ Department of Physics, Mid Sweden University, SE-851 70 Sundsvall, Sweden
}

\begin{abstract}
An important problem in nanotechnology is to develop a method for assembling complex, aperiodic, structures. While simple self-assembly will not be able to address this problem, programmable-, or algorithmic-, self-assembly is powerful enough to be a potential solution. Here, we address the question of how the basic properties of the constituent building blocks are related to the periodicity of the resulting assembly. By introducing the parameters unique structures, which gives a measure of the complexity of an assembly, and bond uniqueness, which gives a measure of how the building blocks fit together, we show how to quantify the structural quality of a general assembly system and present relations between the parameters. The introduced methods will be helpful when designing assembly systems to be used for direct fabrication of nanosystems or for nano-scaffolds and addressable arrays.
\end{abstract}

Keywords: Programmable Self-Assembly, Algorithmic Self-Assembly, Scaffold Assembly, Bond Uniqueness, Tile Systems.

\section{INTRODUCTION}

Probably, the single most important problem in nanotechnology is to develop a method for assembling complex structures, such as a nanochip. Today, in microelectronics different types of lithography is used to define the structures. These methods are referred to as top-down methods. However, the continuing route towards smaller structures using top-down methods is increasingly difficult, which is reflected in fabrication costs that are growing much faster than the electronic market. ${ }^{1}$

A bottom-up building approach based on self-assembly has been widely discussed as an alternative method for nanofabrication. In self-assembly the building blocks, that could be atoms, molecules, or larger structures, diffuse around and eventually bind to a specific location. The simplest type of self-assembly, such as self-assembling monolayers or growth of nanowires, will merely lead to a non-complex crystal, and will be of little use to assemble a chip. We refer to this simplest type of self-assembly as crystal self-assembly (Fig. 1a). At the other extreme are building blocks that all have a unique address tag that will bind to a corresponding address. Using this unique addressing self-assembly method any kind of complex structure might be build, but a large number of building blocks are needed (Fig. 1b). Between these two

*Author to whom correspondence should be addressed. extremes is programmable self-assembly (or algorithmic self-assembly) $)^{2,3}$ (Fig. 1c).

A formal model for studies of programmable selfassembly is the Tile Assembly Model ${ }^{4}$ where the building blocks called tiles are self-assembled into a square lattice. The model is an extension of Wangs theory of tiling, ${ }^{5}$ but each of the sides of the square tiles contains a glue that allow binding of another tiles to the sides. The glue is specific so only a tile-side with the same corresponding glue will bind. Growth starts from a seed tile by adding one tile at the time. By designing the tiles in a specific way the growth can be programmed. Programmable self-assembly is powerful, for example, it has been shown to be capable of universal computation. ${ }^{6}$ The Tile Assembly Model has been used for investigation of, for example, the minimum number of tiles needed to self-assemble a square of a certain size (program size complexity), ${ }^{4}$ the time complexity, ${ }^{7}$ optimal size and time complexity, ${ }^{8}$ or whether a given tile system uniquely produce a given shape. ${ }^{9}$ Furthermore, algorithmic self-assembly systems have been proposed as a tool to study self-replication ${ }^{10}$ in a manner similar to von Neumann self-replicating machines. ${ }^{11}$

Experimentally, DNA double-crossover molecules with four sticky ends, which are analogues to the four sides of the Wang tiles, have been demonstrated to assemble into two-dimensional lattices. ${ }^{12}$ Another experimental demonstration, of a much more complex pattern, is the algorithmic assembly of DNA Sierpinski triangles. ${ }^{2}$ Macroscopic 
systems based on millimeter-scale plastic tiles that float on a liquid ${ }^{13,14}$ can also be used for experimental realization of algorithmic self-assembly. ${ }^{3}$ Recently, DNA-linked nanoparticles has been suggested as a base for programmable self-assembly. ${ }^{15}$ Another path, for doing programmable self-assembly, is to first self-assemble a scaffold of technologically simple building-blocks, then, self-assemble the interesting devices on the addressable sites created by the scaffold assembly. Potential scaffold materials are for example DNA-crystals ${ }^{12,16-19}$ mentioned above or DNA-linked nanoparticle ${ }^{20,21}$ as well as more advanced biological systems like proteins ${ }^{22}$ or viruses. ${ }^{23}$ Using metallization of the biomolecules, the scaffold can also act as an electrical contact between the devices assembled upon the scaffold. ${ }^{24}$ For reviews on some of these self-assembly systems see Refs. [25, 26].

Suppose, that a complex nanosystem, say an electronic chip, is going to be built using programmable self-assembly. From an engineering point of view it is important to know the limits of the assembly system given an available technology. This paper describes a solution to this problem by introducing two parameters: unique structures which gives a measure of how complex the final structure is and the bond uniqueness parameter-which is related to the specificity of the glue or bonds. Together with other building block parameters, such as the number of different types and their total number, we will give limits as well as trade-off relations between different assembly designs. The task of designing an assembly system so that it gives the desired amount of unique structures is discussed in Section 4 where we propose a method for the design of square tile systems.

\section{CLASSES OF SELF-ASSEMBLY}

In the following we use the word tile to denote a general assembly building block. A tile have one or more functional edges with specific types of glues, or bonds.

Depending on the types of open bonds on the selfassembly tiles and on the physical process that makes the tiles assemble, we classify the process according to the type of assembly it can produce.

In Crystal self-assembly (Fig. 1(a)) patterns of tiles are reproduced throughout the assembly. Since a crystal selfassembly (CA) repeats itself, the neighborhood of each tile must be identical everywhere in the assembly. This leads to the conclusion that each open bond of a crystal tile can only make a bond with one specific type of tile. This constraint on the assembly process assures that each tile of a certain type has the same type of tile neighbors everywhere.

In a unique addressing assembly (UA) (Fig. 1(b)), each tile type only occurs once and the tile position is completely defined. ${ }^{4}$ Each bond must be specific as to what type of tile it can accept, like in the CA case above. There (a) CA-Crystal Assembly
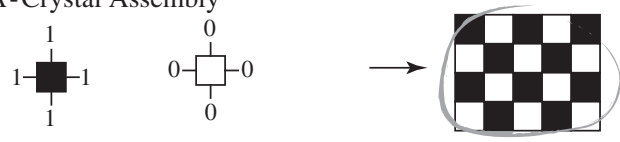

(b) UA - Unique Addressing assembly

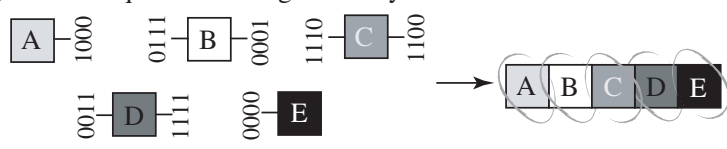

(c) PSA - Programmable Self-Assembly

$\longrightarrow$

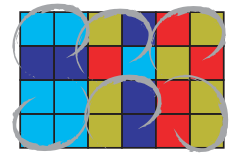

Fig. 1. Classes of self-assembly systems and unique structures (encircled in gray) in generated assembly patterns. (a) Type 1 bonds can connect to type 0 bonds. Each of the tiles bonds can only connect to one other type of tile. This leads to crystal type assembly. The only unique structure is the entire assembly. (b) Each tile has bonds that can connect only to one other type of tile, and each tile has no more than one type of each bond. The resulting assembly type is unique addressing where each tile constitutes a unique structure. (c) In this case each bond can host two different types of tiles. Together with the added criterion that each incoming tile must interact with at least two other tiles in order to assemble, the process is now of the PSA class. This assembly process needs to be nucleated for the assembly to grow. The resulting assembly in (c) has six unique structures of 4 tiles each $(S=6, \eta=4)$.

must also exist a unique way to arrange the tiles so that there are no more open bonds or so that the remaining open bonds are blocked by the assembly itself.

A Programmable Self-Assembly (Fig. 1(c)) (PSA) system produces assemblies where one can find both repeating and unique patterns of tiles. For this to happen, some, or all, of the open bonds of a PSA tile must be able to bond to more than one type of tiles. Since an assembled PSA tile must, according to the above definition, accept at least two different types of new neighbors the process is not deterministic and thus not programmable; a single PSA tile alone cannot uniquely define the next tile in the assembly. At least one second neighboring, PSA tile needs to be present to uniquely determine the next tile type. One thus introduce the added criterion that a new tile must bind to at least two, already assembled, tiles; i.e., two tiles is the smallest structure that define the type of the third tile to be assembled. We do not know if this is generally true that programmable semi repetitive systems need this assembly criteria. This feature seems to be the single most important factor for the creation of complex assemblies. In nature, this type of dependent binding has been shown to be an important feature. For example in the self assembly of ribosomes certain proteins do only assemble if the growing ribosome assembly contains a certain pair of previously assembled proteins. ${ }^{27}$

Winfree and Rothemund has made a similar observation in discussions of the need for cooperation in order 
for the assembly process to be programmable. ${ }^{4}$ Klavins et al. $^{28}$ has proved that no binary grammar can generate a unique stable assembly, however, this proof is valid for self-assembly systems with conformal switching tiles, i.e., building blocks that change state after being assembled.

\section{UNIQUE STRUCTURES AND BOND UNIQUENESS}

As noted in the introductory discussion above, one issue that makes self-assembly interesting for nanotechnology is the ability to construct scaffolds with uniquely addressable structures. In the following we will show how it is possible to estimate the number of unique structures that a certain assembly system can produce.

In each finite assembly, one can identify a certain number of unique structures, $S$. A unique structure is a number of connected tiles that is not reproduced anywhere else in the assembly. As an example, the string ac is a unique structure in aaabacabca while $a b$ is not. The minimum number of tiles needed to define a unique structure is denoted $\eta$ or, number of tiles per unique structure. The symbol $N$ will be used to specify the total number of tiles that constitute the assembly in the discussion that follows. The number of tile types will be denoted by $w$.

The patterns in Figure 1 are examples of a few simple cases where finding $S$ and $\eta$ is straightforward. In general it is always possible to divide an assembly into $S$ unique structures of $\eta$ tiles in each structure. The pair $(S, \eta)$ is defined to be; the maximum possible $S$, and the $\eta$ that gives this maximum. A unique structure parser algorithm for finding the values of the pair $\eta$ and $S$ in any finite assembly, can be constructed. Note that unique structures may not overlap. This way, each unique structure can be interpreted as an address in a scaffold, to be used for subsequent assembly.

Consider the task of assembling a linear structure of $S$ addressable locations. Since each location should be uniquely addressable, the structure constituting the location must be globally unique. One way to proceed is to make exactly $S$ tiles that stick together in only one way (unique addressing, see Fig. 2(a)), or to make some number $w$ of unique tiles where $w<S$ (PSA). If one chooses the later strategy one must figure out a clever way to make the $w$ tile types assemble into $S$ unique structures. Two examples of model PSA systems are found in Figures 2(b) and (c). The prime-tower assembly (Fig. 2(b)) counts to a product of two prime numbers and then stops the assembly. This type of process is similar to the vernier process in biology, that is believed to be a length controlling mechanism in linear protein assembly. ${ }^{29}$ The counter by Cheng, Goel, and Moisset de Espanés (CGM $)^{8}$ is an assembly counter that is optimized to use as few tile-types as possible (optimized for low $w$ ) and still be able to produce arbitrarily long sequences. Each row is $\log _{2} S$ tiles wide and constitutes a unique structure and $w=8$.

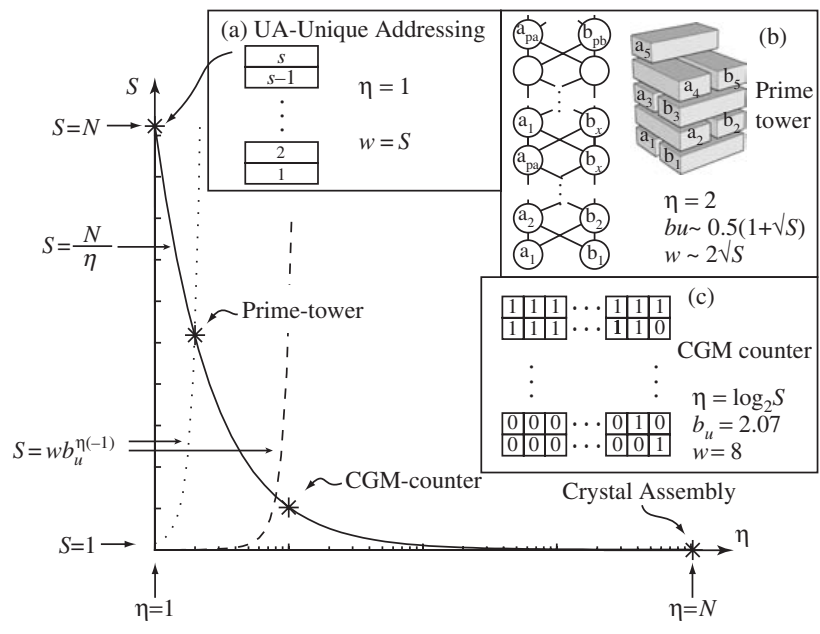

Fig. 2. A plot of $S$ versus $\eta$ for different kind of linear-, counter type-, assemblies at constant number of total tiles, $N$. The $\eta$-axis is logarithmic. The solid line represents the bound $S=N / \eta$. The insets (a)-(c) show model systems of linear assembly displaying $S$ unique structures. The process in (a) is an example of a one-dimensional UA assembly. (b) Shows a modulo-prime counter. Two sets of tiles, $a$ 's and $b$ 's, consisting of $p_{a}$ and $p_{b}$ tiles each ( $p_{a, b}$ are prime numbers). The assembly stops when tiles $a_{p_{a}}$ and $b_{p_{b}}$ are next to one another. To the right: physical model of the assembly. Left: a graph displaying the bond structure. In (c) the counter is of the type described in Ref. [8] (referred to as CGM-counter in this paper). This is a pseudo binary counter under the tile assembly model ${ }^{4}$ that uses 8 distinct tiles. In the described model systems each row constitutes a unique structure. The prime number counter have $\eta=2$ and $S \sim N / 2$. The CGM counter have $\eta=\log _{2} S$ where $S$ is determined from the equation $S=N / \log _{2} S$. The dotted and the dashed lines are Eq. (2) written with the values of $w$ and $b_{u}$ for the prime-counter and the CGM counter, respectively. (The graph is plotted for $N=100$.)

When the total number of tiles, $N$, is constant, it is possible to draw some conclusions about $S$ and $\eta$ for several types of assembly processes. The simplest cases being unique addressing, where $S$ would be equal to $N$ and $\eta=1$, and crystal type assembly, where $S$ would be 1 and $\eta=N$. Programmable self assembly lies somewhere in between. We start the discussion by considering the number of possible ways one may assemble a substructure containing $\eta$ tiles.

If the assembly is random, the number of ways that one may combine $\eta$ tiles chosen from $w$ types is given by $w^{\eta}$. PSA processes are not random however, and the number of ways one may combine tiles into structures of $\eta$ tiles is limited by the bond uniqueness of the assembly system.

The bond uniqueness, $b_{u}$, for an assembly system is defined as the average number of tile types that each open bond can bind to. For example in the assembly system in Figure 1(c) the bond uniqueness is equal to two because each specific open bond can only harbor two different tile types on average. If the number of bonds per tile is denoted $k(k=4$ for the square-tile systems discussed below, since each tile has four bonds), the total number of bonds on all the tile types is $w k$. Each bond on each tile type is given an index $i$. The bond uniqueness is then calculated in the 
following way:

$$
b_{u}=\frac{\sum_{i}^{k w} \text { No. of tile types that bond } i \text { can bind to }}{k w}
$$

Returning now to the construction of structures containing $\eta$ tiles. The first tile in such a structure can be chosen at will from the $w$ available tile types. Once this tile is specified however, the following tile types to be placed next to the original tile, can only be chosen from a subset of the tile types, containing $b_{u}$ tiles. So a row ( $\eta$ tiles wide) in a linear-, counter type-, assembly can be constructed in $w b_{u}^{\eta-1}$ number of ways. If each row is to constitute a unique structure we get that the maximum number of rows, i.e., the maximum $S$ for the given $\eta$ is:

$$
S=w b_{u}^{\eta-1}
$$

This equation, together with $N=S \eta$, gives the number of unique structures for linear-, counter type-, assemblies (see Fig. 2).

In two dimensional assembly we consider each unique structure to be a square of $\eta$ tiles. Once the edge is specified, in the form of an L, the interior is uniquely determined by the assembly system. The corner tile can be chosen among all $w$ tile types and each arm of the Lshaped edge can be chosen in $b_{u}^{\left(\eta^{1 / 2}-2\right)}$ ways (the structures are assumed to be square and the edges thus $\eta^{1 / 2}$ tiles long). In general we get that the number of possible ways to build structures containing $\eta$ tiles is given by the following expression:

$$
\text { No. of possible } \eta \text {-structures }=w b_{u}^{\left(\eta^{1 / D}-D\right)}
$$

where $D$ is a dimensionality constant that is equal to 2 or 3 depending on whether the assembly is two-dimensional or three-dimensional.

Consider a two dimensional assembly with $N$ tiles (assume that it is $\sqrt{N} \times \sqrt{N}$ tiles big). There are $(\sqrt{(N)}-$ $\sqrt{\eta}+1)^{2}$ locations where a $\sqrt{\eta} \times \sqrt{\eta}$-tiles structure may be located (result from 2D-word pattern matching see for example Ref. [30]). Assume that each one of these structures must be unique and that we use the maximum number of variations of $\eta$-structures that the tile system may produce. Then from Eq. (3) we get that

$$
\left.w b_{u}^{\left(2 \eta^{1 / 2}-2\right)}=(\sqrt{(} N)-\sqrt{\eta}+1\right)^{2}
$$

And in general

$$
w b_{u}^{\left(D \eta^{1 / D}-D\right)}=\left(N^{1 / D}-\eta^{1 / D}+1\right)^{D}
$$

Now note again that, since the $S$ unique structures that we seek may not overlap, the total number of tiles is $\eta$ taken $S$ times:

$$
N=S \eta
$$

Using (6) to solve for $S$ in (5), and taking logarithms we get the following result:

$$
S=N\left(\frac{D \log b_{u}}{\log \left[\frac{b_{u}^{D}}{w}\left(\left(N^{1 / D}-\left(\frac{N}{S}\right)^{1 / D}+1\right)^{D}\right)\right]}\right)^{D}
$$

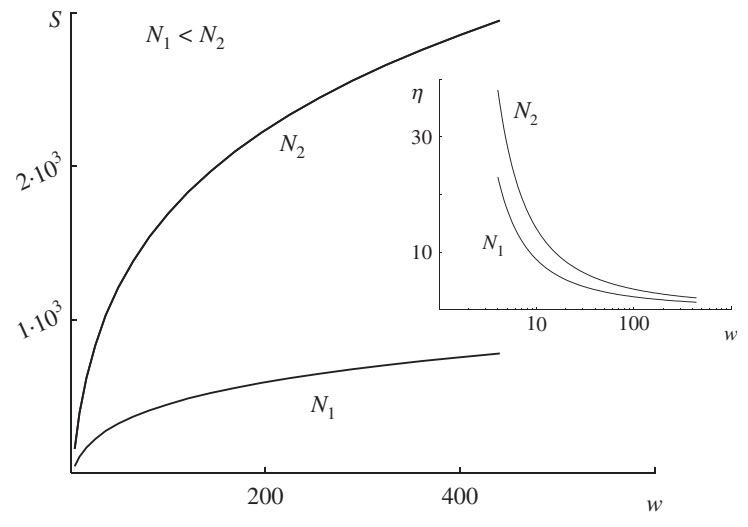

Fig. 3. Characteristics resulting form Eq. (7) showing the number of unique structures, $S$, in a two dimensional assembly plotted as a function of the number of tile types, $w . b_{u}=\sqrt{w}$ is assumed. Plotted for two values of total number of tiles $N$ where $N_{1}=1000$ and $N_{2}=6000$. As the number of tile types is increased the growth of $S$ slows down. The inset shows the same curves but instead of $S$ we plot the size of the unique structures, $\eta$, on the $y$-axis (where $\eta=N / S$ ). The $w$-axis is logarithmic in this case. As $w$ is increased the size of the unique structures rapidly decreases.

Equation (7) gives the maximum number of unique structures of a PSA process of $N$ total tiles with $w$ tile types and bond uniqueness $b_{u}$. This relation gives an optimal number of unique structures, in practice, the value of $S$ could be less than this depending on the periodicity of the seed or nucleation process used. In Figure 3 a graphical interpretation of (7) is given.

\subsection{Example of Application}

The results above will be helpful when designing nanosystems using self-assembly. Consider an example where one wants to construct a scaffold for nanodevices. Lets say that we have the technology to produce 9 types of building blocks that each measures $13 \mathrm{~nm} \times 13 \mathrm{~nm}$. They have $b_{u}=3$. How many uniquely addressable structures can we optimally produce in an area measuring $1 \mu \mathrm{m} \times$ $1 \mu \mathrm{m}$. The total number of tiles we have room for is thus $N \approx 5900$. Using Eq. (7) with $D=2$, and solving numerically, we get that the maximum number of unique structures is approximately 380 so $\eta=N / S \approx 15.5$. It should thus be possible to construct a scaffold comprising some 370 addressable sites, each containing 16 tiles. (If one uses $\eta=15$, which is less than the estimated value, the assembly system will not be able to cover the entire area with unique structures, some will necessarily be repeated.) The unique structures/addressable locations are thus in the order of $50 \mathrm{~nm} \times 50 \mathrm{~nm}$. If one wishes to make them smaller, more tile types are needed.

\section{DESIGN OF A SELF-ASSEMBLY SYSTEM}

Once the important design parameters are fixed, how do one proceed with the actual design of the building blocks? We assume that the values of $w$ (number of tile types) and 
$b_{u}$ (bond uniqueness) have been determined to provide the desired amount non-periodicity in the assembly by using Eq. (7) (i.e., the desired amount of unique structures). Lets consider the case where a two-dimensional assembly is constructed from square tiles, each of them having four bonds to assemble into a square lattice. Similar schemes as the one presented here may be used for any type of planefilling, or space-filling tiles. The bonds are considered to be of a complimentary nature so that a bond may not bind to a bond of the same type but only to its complement. Examples of complementary bond types are DNA-strands and jigsaw-puzzle pieces.

The tiles can be made non-rotatable by dividing the bonds into north-south and east-west pairs. ${ }^{\dagger}$ North-bonds must thus be complementary to south-bonds and eastbonds complementary to west-bonds. We denote by $n_{\mathrm{NS}}$ and $n_{\mathrm{EW}}$ the number of bond-pairs that are of the northsouth-, and east-west-type, respectively. So, the total number of bonds of the south type for example is $n_{\mathrm{NS}}$, of the north type $n_{\mathrm{NS}}$, and together they form $n_{\mathrm{NS}}$ complementary pairs. Assembly growth is possible in any direction.

Remember that the bond uniqueness is the average number of tile-types that each bond can bind to. Now we know that the number of south bonds is $n_{\mathrm{NS}}$, the number of tiletypes is $w$, so each type of south bond can be found on $w / n_{\mathrm{NS}}$ tile types on average. By consequence, the north bonds will have an average bond uniqueness of $w / n_{\mathrm{NS}}$. By the same argument the south bonds will have an average $b_{u}$ of $w / n_{\mathrm{NS}}$ and the east and west bonds an average $b_{u}$ of $w / n_{\mathrm{EW}}$ each. The total bond uniqueness is the average over the four E-W-S-N bond classes:

$$
b_{u}=\frac{w}{2}\left(\frac{1}{n_{\mathrm{NS}}}+\frac{1}{n_{\mathrm{EW}}}\right)
$$

To relate the values of $n_{\mathrm{NS}}$ and $n_{\mathrm{EW}}$ with the number of tile types, $w$, we will now consider the number of possibilities to construct corner sites. A corner site is a site where programmable self-assembly can occur, i.e., a site in the assembly where an incoming tile may bind to at least two other assembled tiles. In this discussion we consider square, two-dimensional tiles, so the corner sites are L-shaped trimers, like the one in Figure 4. The bottom left tile can be chosen at will from the $w$ tiles whereas the tiles to the north and east of this tile can be chosen in $w / n_{\mathrm{NS}}$ or $w / n_{\mathrm{EW}}$ ways, respectively. So the total number of possible L-shaped trimers is $w^{3} /\left(n_{\mathrm{NS}} n_{\mathrm{EW}}\right)$. Looking now at an individual tile, $A$, the number of $\mathrm{L}$-shaped trimers that can be created by starting out from tile $\mathrm{A}$ is determined by

\footnotetext{
${ }^{\dagger}$ Note that the tiles are still physically rotatable, but by the way we organize the bond-pairs the tiles will be forced to line up with the rest of the assembly so that every copy of a tile will always have the same rotation.

${ }^{\ddagger}$ The choice of direction of the trimer construction is arbitrary and the results will be the same if one considers L-shaped trimers with different rotations.
}

(a)

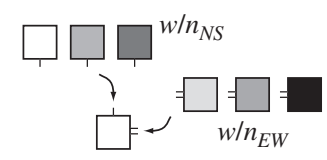

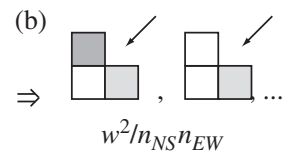

Fig. 4. (a) Each open north-, or east-bond can bind to $w / n_{N S}$ or $w / n_{E W}$ tile types on average. This creates on average, for each starting tile, $w^{2} /\left(n_{N S} n_{E W}\right)$ possible corners like the one marked by an arrow in (b). Each of these corner sites must uniquely define the tile type that should bind to the site. If the number of created corners is greater than $w$ for some tile then one tile type must fit two of the created corner sites. This violates the principle of programmable self-assembly and would yield a non-deterministic assembly process.

the bond uniqueness of the bonds of tile A. One thing is certain however, tile A must not be able to create more corner sites than there are tile types. If this would be the case then at least one of the tile types would have to fit more than one of the created corner sites. This would in turn violate the principle of programmable self-assembly that each incoming tile is uniquely determined by binding to at least two bonds. Thus we conclude that the number of corner sites each individual tile may create must be less than or equal to $w$. The average number of L-shaped trimers each tile may create is $\frac{w^{3}}{n_{\mathrm{NS}} n_{\mathrm{EW}}} / w$, so $w^{2} /\left(n_{\mathrm{NS}} n_{\mathrm{EW}}\right) \leq w$ following the above arguments. However, if one tile generates less than $w$ corner sites, then another tile must generate more than $w$ corner sites in order for the total number of corner sites to be correct. As mentioned, this is not allowed and the only possibility left is thus

$$
\frac{w^{2}}{n_{\mathrm{NS}} n_{\mathrm{EW}}}=w \Rightarrow w=n_{\mathrm{NS}} n_{\mathrm{EW}}
$$

The only exception to this is when $b_{u}=1$, i.e., when the assembly is of the crystal type and not of the PSA type, then each tile can generate exactly one corner site and $n_{\mathrm{NS}}=n_{\mathrm{EW}}=w$.

The Eqs. (8) and (9) constitute an equation system with the following solution:

$$
n_{\mathrm{NS}, \mathrm{EW}}=b_{u} \pm \sqrt{b_{u}^{2}-w}
$$

where $n_{\mathrm{NS}}, n_{\mathrm{EW}}$, and $w$ must be positive integers. Since all the numbers in (10) are real, we get the following constraint on the values of the bond uniqueness

$$
b_{u}^{2} \geq w
$$

\subsection{Designing Tiles from Corners}

Following the results above we can give a general method for designing square, non-rotating, tile systems having a certain number of tile types and bond uniqueness. The method can be extended to non-rotating tile types of other geometries.

A corner is defined as a pair of two adjacent bonds on a tile. Square tiles will have four corners, i.e., four pairs of 
(a)

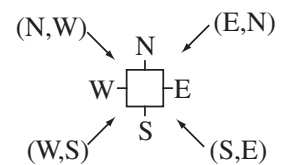

(b)

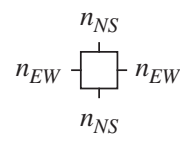

Fig. 5. (a) The corners of a square tile. (b) The number of choices for the types of bonds is $n_{\mathrm{NS}}$ for the north and south bonds and $n_{\mathrm{EW}}$ for the east and west bonds. Since each pair of bonds at a corner must be unique, $w \leq n_{\mathrm{NS}} n_{\mathrm{EW}}$.

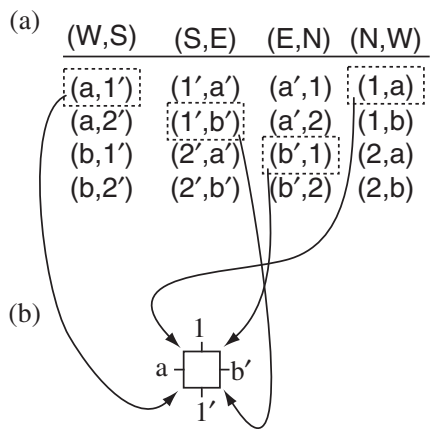

$$
\begin{aligned}
& \text { (c) } \\
& n_{N S}=n_{E W}=2 \\
& b_{u}=2 \\
& w=4
\end{aligned}
$$

Fig. 6. Example showing the construction of a tile-system with $n_{\mathrm{NS}}=$ $n_{\mathrm{EW}}=2$. Primed bonds fit the unprimed counterpart. $\mathrm{a}, \mathrm{b}$ are west bonds, $\mathrm{a}^{\prime}, \mathrm{b}^{\prime}$ east bonds, and 1, 2 and $1^{\prime}, 2^{\prime}$ north and south bonds, respectively. (a) A table showing the total number of possible WS, SE, EN, and NW bond-pairs. (b) Four corner bond-pairs makes one tile. (c) Proceeding in the same manner as in (b) generates a complete tile-system with $w=4$ and $b_{u}=2$. (This is the same system as in Fig. 1(c)).

bonds: (W, S), (S, E), (E, N), and (N, W), ${ }^{\mathbb{4}}$ see Figure 5(a). Hexagonal tiles will have six corners and cubes in threedimensional assembly will have eight corners where each corner will be a bond triplet.

When the number of bondtypes, $n_{\mathrm{NS}}$ and $n_{\mathrm{EW}}$, have been decided, one may construct $n_{\mathrm{NS}} n_{\mathrm{EW}}$ corner pairs for each type of corner (WS, SE, EN, and NW), in total $4 n_{\mathrm{NS}} n_{\mathrm{EW}}$ pairs. To construct the tiles, simply pair the corners four and four, see Figure 6 for an example. Because Eq. (9) holds, all corner pairs must be used, and each corner pair must only be used once. If one corner pair were to occur on two tiles, the corresponding corner site would not uniquely define a single tile and the assembly would be random.

\subsection{Example}

The proposed method for tile design should be proceeded with a check for the technology limits in order to see that the desired requirements can be met. The following is an example of such a check:

Suppose we have a technology to produce $10 \mathrm{~nm} \times$ $10 \mathrm{~nm}$ square tiles using a maximum of 14 bond pairs (suppose for example that we only have 28 DNA-strands to work with). So $n_{\mathrm{NS}}+n_{\mathrm{EW}}=14$. Is it possible to cover a surface of $1 \mu \mathrm{m} \times 1 \mu \mathrm{m}$ and get uniquely addressable structures that are maximum 4 tiles big $(\eta=4, S=2500$, $N=10000)$ ? By combining Eqs. (8) and (9) we find that

\footnotetext{
"The notation assumes a counter-clockwise walk around a tile, this is why we use WS and EN instead of SW and NE.
}

$b_{u}=\frac{1}{2}\left(n_{\mathrm{NS}}+n_{\mathrm{EW}}\right)$, so $b_{u} \leq 7$. Since $w=n_{\mathrm{NS}} n_{\mathrm{EW}}$ we know that the maximum number of tile types is $w=49$. Using Eq. (7) with $D=2$ we get that the maximum number of unique structures that we can expect is $S=1796$ so the answer is no, we would need more bond types.

\section{DISCUSSION}

Depending on the application, structures that show repetitions if rotated may, or may not, count as unique. If we do not regard structures, that show repetitions while rotated, as unique, then we must divide the total number of unique structures by the rotational symmetry of the lattice. In the case of square tiles the factor is four. If one considers the case of constructing nanosystems using assembly of nanodevices the orientation of the nanodevices will often be important and we should thus take this factor into consideration.

Another important issue that will affect the assembled structure is the relative concentrations of the tile-types. In Eq. (7) we assume that the relative concentrations are all equal $1 / w$. Any deviation from this will lead to a reduced number of unique structures.

Looking now at the error rate in self-assembly, how is it related to the bond uniqueness? At thermal equilibrium the probability of forming a certain configuration $L$ is given by: ${ }^{31}$

$$
\mathscr{P}_{L}=\frac{\exp \left(-U_{L} / k_{B} T\right)}{\sum_{L_{i}} \exp \left(-U_{L} / k_{B} T\right)}
$$

where $U_{L}$ is the energy of the formation, $T$ is the temperature, and $k_{B}$ is Boltzmanns constant. Assuming that the energy of all bonds are equally strong and the binding energy is equal to $U$. Assuming that the correct binding of a tile will have binding energy $2 U$ (two bonds fit) and any incorrectly bonded tile (only one bond fit) will have energy $U$. For each open bond in an assembly there are $b_{u}$ tiles that may bind to that bond but only one of these tiles is the correct one. Consequently, for a corner site with two open bonds there are $2\left(b_{u}-1\right)$ possible ways to add an incorrect tile type by making a single bond. Following this discussion, we get that the probability of a correct assembly event is given by:

$$
\begin{aligned}
\mathscr{P}_{L} & =\frac{\exp \left(-2 U / k_{B} T\right)}{\exp \left(-2 U / k_{B} T\right)+2\left(b_{u}-1\right) \exp \left(-U / k_{B} T\right)} \\
& \simeq 1-2\left(b_{u}-1\right) \exp \left(U / k_{B} T\right)
\end{aligned}
$$

The complimentary probability is the probability of errors and as shown in Eq. (13) it is proportional to $b_{u}$. If one is concerned about the correctness of the assembled structures it is thus advisable to choose a low value for the bond uniqueness.

Since we have found that, for square tile systems that are made non-rotating, $b_{u} \geq w$, we can also conclude that the error rate is at best proportional to $\sqrt{w}$. Making complex assemblies with spatially small unique structures (low $\eta$, high $w$ ) might therefore be harder than previously 
assumed. Proposed methods for error correction exists ${ }^{32}$ but since they rely on subdivision of the tiles, in effect creating even more tiles per unique structure, one needs to take this into consideration when evaluating the size of the unique structures. The results in this paper are still valid, the extra tiles needed for error correction is simply a multiplication factor of the number of tile types needed and of the resulting number of tiles per unique structure.

\section{CONCLUSIONS}

We have presented a method for the parametrization of assembly systems derived from their ability to form unique structures. We have also introduced the concept of bond uniqueness and showed how it influences the number of unique structures that a programmable self-assembly system can create. The structural complexity is heavily dependent on the bond uniqueness of the system. By using the relations obtained in this paper (mainly Eq. (7)) for nanotechnology applications it will possible to vary the parameters $w$ (number of types of building blocks) and $b_{u}$ (their bond uniqueness) to obtain the desired structural complexity and the desired size $(\eta)$ of the addressable locations/unique structures. In Section 4 we showed how these parameters influences the number of bond types required, and the design of the actual building blocks to be used for the assembly. The introduced concepts will prove helpful when designing tile systems and evaluating the theoretical limits of a proposed self-assembly technology at an early stage.

\section{References}

1. R. Compañó (ed.), Technology Roadmap for Nanoelectronics, European Commission, Office for Official Publications of the European Communities, Luxemburg (2001).

2. P. W. K. Rothemund, N. Papadakis, and E. Winfree, Plos Biology 2, 2041 (2004)

3. P. W. K. Rothemund, Proc. Nat. Acad. Sci. 97, 984 (2000).

4. P. W. K. Rothemund and E. Winfree, in Proc. 32nd Ann. ACM Symp. Theor. Comp. New York, NY and Portland, OR, USA (2000), p. 459.

5. H. Wang, Bell Sys. Tech. J. 40, 1 (1961).

6. E. Winfree, in DNA Based Computers, DIMACS series in Discrete Math. and Theor. Comp. Sci., edited by R. J. Lipton and E. B. Baum, American Mathematical Society, Princeton (1996), Vol. 27, p. 199.
7. L. M. Adleman, Q. Cheng, A. Goel, and M. D. Huang, ACM Symposium on Theory of Computing (STOC'01) (2001), p. 740.

8. Q. Cheng, A. Goel, and P. Moisset de Espanés, in Proceedings of Foundation of Nanoscience: Self-Assembled Architectures and Devices, Sciencetechnica, Snowbird, Utah (2004).

9. L. M. Adleman, Q. Cheng, A. Goel, M. D. Huang, D. Kempe, P. Moisset de Espanés, and P. W. K. Rothemund, ACM Symposium on Theory of Computing (STOC'02) (2002), p. 23.

10. R. Schulman and E. Winfree, in Advances in Artifical Life, Proc., Lect. Notes Artif. Intel. (2005), Vol. 3630, p. 734.

11. D. Mange, A. Stauffer, L. Peparolo, and G. Tempesti, Proc. IEEE 92, 1929 (2004).

12. E. Winfree, F. R. Liu, L. A. Wenzler, and N. C. Seeman, Nature 394, 539 (1998).

13. K. Hosokawa, I. Shimoyama, and H. Miura, Sens. Actuators A: Phys. 57, 117 (1996).

14. N. Bowden, A. Terfort, J. Carbeck, and G. M. Whitesides, Science 276, 233 (1997).

15. B. Högberg, J. Liu-Helmersson, S. Holm, and H. Olin, Appl. Surf. Sci. (2006), in press.

16. S. H. Park, H. Yan, J. H. Reif, T. H. LaBean, and G. Finkelstein, Nanotechnology 15, 525 (2004).

17. D. Dwyer, S. H. Park, T. LaBean, and A. Lebeck, in Proceedings of the 2nd Conference on the Foundations of Nanoscience, Sciencetechnica, Snowbird, Utah (2005), p. 187.

18. S. H. Park, C. Pistol, S. J. Ahn, J. H. Reif, A. R. Lebeck, C. Dwyer, and T. H. LaBean, Angew. Chemie Int. Ed. 45, 735 (2006).

19. Y. Y. Pinto, J. D. Le, N. C. Seeman, K. Musier-Forsyth, T. A. Taton, and R. A. Kiehl, Nano Lett. 5, 2399 (2005).

20. A. P. Alivisatos, K. P. Johnsson, X. G. Peng, T. E. Wilson, C. J. Loweth, M. P. Bruchez, and P. G. Schultz, Nature 382, 609 (1996).

21. C. A. Mirkin, R. L. Letsinger, R. C. Mucic, and J. J. Storhoff, Nature 382, 607 (1996).

22. Shuguang Zhang, Biotechnol. Adv. 20, 321 (2002).

23. A. S. Blum, C. M. Soto, C. D. Wilson, J. D. Cole, M. Kim, B. Gnade, A. Chatterji, W. F. Ochoa, T. W. Lin, J. E. Johnson, and B. R. Ratna, Nano Lett. 4, 867 (2004).

24. H. Yan, S. H. Park, G. Finkelstein, J. H. Reif, and T. H. LaBean, Science 301, 1882 (2003).

25. G. M. Whitesides and B. Grzybowski, Science 295, 2418 (2002).

26. N. C. Seeman, Nature 421, 427 (2003).

27. G. M. Culver, Biopolymers 68, 234 (2003).

28. E. Klavins, IEEE Proc. Int. Conf. Robotics and Automation 5, 5293 (2004).

29. J. S. Lindsey, New J. Chem. 15, 153 (1991).

30. A. Carpi and A. de Luca, Int. J. Found. Comp. Sci. 15, 355 (2004).

31. B. N. Roy, Fundamentals of Classical and Statistical Thermodynamics, Wiley, West Sussex, England (2002).

32. E. Winfree and R. Bekbolatov, Lect. Notes Comp. Sci., DNA Computers 9,126 (2004).

Received: 27 February 2006. Accepted: 9 March 2006. 